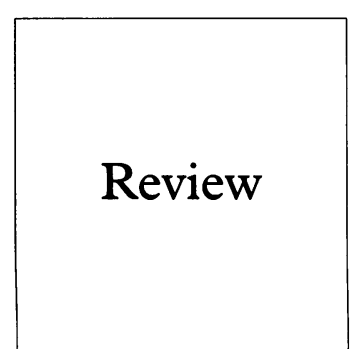

Cytomegalovirus (CMV) infection is a very common opportunistic manifestation of HIV disease ranging from $10 \%$ to $40 \%$ during AIDS. ${ }^{12}$ CMV retinitis is the most common manifestation.

From 1991 to 1996, CMV infection had been diagnosed in 632/1971 (32\%) AIDS patients in our unit, $65 \%$ of them having retinitis. After AIDS diagnosis, the risk of CMV visceral manifestations was $16 \%$ at 1 year, $34 \%$ at 2 years, and $46 \%$ at 3 years. The risk of CMV has increased over time owing to both a longer survival induced by antiretroviral therapies and a better management of opportunistic infections in patients with a persistent low immune status. In a natural history study of 1002 patients the probability of CMV disease at 2 years was $21.4 \%$ for patients with levels of CD 4 below $100 \times 10^{6} / 1$ compared with $10 \cdot 3 \%$ for those with $\mathrm{CD} 4$ greater than $100 \times$ $10^{6} / 1 .^{1}$ Interestingly "the protease inhibitors era" which started in the USA and some European countries, in spring 1996 has dramatically changed the clinical spectrum of HIV infection. Approximately $50-80 \%$ of patients receiving highly potent antiretroviral therapy including protease inhibitors exhibit a median decrease of 1 to $2 \log _{10}$ HIV RNA viral load with $60-80 \%$ of them going below detectable level, with a significant increase in CD4 lymphocytes even when initially they have a very low immune status. This had led to a dramatic decrease in different clinical syndromes related to HIV, such as wasting syndrome, cryptosporidiosis, microsporidiosis, or atypical mycobacterial infection. The consequences of highly active antiviral therapy (HAART) on CMV infection have not yet been fully established; however, in our experience, the incidence of CMV disease has decreased from 24/100 patients a year to $18 / 100$ patients a year in 1996 (C Katlama, unpublished data).Therefore, it is reasonable to expect that better treatment of HIV infection, allowing control of HIV replication and thus an improved stability in immunological status with delay in the incidence and the time of occurrence of CMV infection.

\section{Diagnosis of CMV retinitis}

CIRCUMSTANCES OF DIAGNOSIS OF CMV RETINITIS

Diagnosis of CMV retinitis is based on clinical findings and may be made in different circumstances.

\section{Presence of visual symptoms}

CMV retinitis commonly induces symptoms such as visual blur, floaters, and loss of peripheral vision which should lead to a full fundus- copic examination. CMV retinitis is not associated with pain, redness of eye, or photophobia unless associated with anterior uveitis.

\section{Systematic funduscopy}

In patients with low immune status (CD4 lymphocytes below 100 or $\left.50 \times 10^{6} / 1\right)$ it should be common practice to regularly examine the retina through funduscopic examination which may reveal anterior retinitis. CMV retinitis can be detected by systematic funduscopy performed in a context of other manifestations or disease-for example, colitis or CMV visceral localisations.

\section{CMV reactivation markers}

These markers of CMV reactivation are strong predictors of development of CMV infection including retinitis. In a prospective study, it has been shown that $50 \%$ of patients with a positive CMV viraemia at the time of AIDS diagnosis will develop a CMV disease within a period of 8 months compared with $11 \%$ of those with a negative viraemia within a mean period of 11 months. ${ }^{3}$ Furthermore, in a multivariate analysis, the presence of positive viraemia had a positive predictive value seven times higher in predicting the risk of further development of CMV focal disease compared with the absolute number of CD4 lymphocytes. Several studies indicate that approximately $50 \%$ of patients who were polymerase chain reaction (PCR) CMV DNA positive will develop CMV disease. ${ }^{4-6}$

\section{DIAGNOSTIC CRITERIA FOR CMV RETINITIS}

Owing to the impracticality of obtaining retinal tissue for histopathological examination, the diagnosis of CMV retinitis is made on the appearance of the characteristic perivascular fluffy yellow-white retinal infiltrates often associated with retinal haemorrhages. In some circumstances, lesions may be more granular rather than fluffy in appearance. Progression of retinitis occurs from the periphery to the central part of retina with an agranular, white leading edge leaving behind an atrophic scar. The location of lesions has important consequences for vision. Retinitis localised in the immediate vicinity of the macula (zone 1) can be rapidly sight threatening and should lead to immediate initiation of treatment. In contrast, lesions outside major vascular vessels (zone 2 or 3), commonly referred to as "peripheral retinitis", are not immediately sight threatening even when the treatment is deferred for a few days. An important point to keep in mind is the fact that "destroyed retinal tissue" cannot be restored and remains as scarring. Furthermore, active CMV proliferation during maintenance therapy is only slightly impaired, and 
frequently reactivates. Many factors may account for this including poor penetration into the eye, too low dosages of anti-CMV drugs, or reduced sensitivity of the virus to the drugs. Therefore, once settled, the CMV necrotic process is almost never arrested but is characterised by successive reactivations followed by residual necrotic retinitis after several acute phases, with subsequent progressive loss of vision.

CMV retinitis is characterised by a minimal vitreal and anterior chamber inflammation. Retinal lesions, at an early stage, may be confused with other ocular lesions such as cotton wool spots which are common in HIV patients, but these are not progressive on serial funduscopic examination. Other agents directly causing retinal necrosis, and less common than CMV, include herpes zoster or herpes simplex infections, which have a rapid course, or toxoplasmic choroidoretinitis which is accompanied by a more pronounced inflammation.

\section{Treatment of CMV retinitis}

\section{ANTIVIRAL DRUGS ACTIVE AGAINST CMV}

Foscarnet

Foscarnet is a pyrophosphate analogue which directly inhibits viral DNA polymerase without the need for prior phosphorylation to be active. It has a broad antiviral spectrum ${ }^{7}$ and is active against ganciclovir resistant $\mathrm{CMV}$ strains, herpes simplex (HSV) including aciclovir resistant HSV strains, and varicella zoster virus (VZV). It has potential activity against $\mathrm{HHV} 8$, the putative agent for Kaposi's sarcoma. ${ }^{8}$

Foscarnet is given by intravenous daily infusion-90 $\mathrm{mg} / \mathrm{kg}$ twice daily as induction therapy for 2 to 3 weeks, and $90-120 \mathrm{mg} / \mathrm{kg} /$ day as one daily infusion in maintenance therapy. ${ }^{910}$ Concomitant hydration with isotonic saline has considerably reduced the incidence of renal toxicity which represents the major adverse event of this therapy. ${ }^{11}$ Evaluation of oral hydration is ongoing but preliminary results during induction therapy suggest that oral hydration can be safely used instead of intravenous hydration. ${ }^{12}$ Other side effects include hypocalcaemia, genital ulcerations, and nausea/vomiting.

Foscarnet offers the advantage of being active on ganciclovir resistant strains, and it has an anti-HIV activity with an average 0.3 to $0.5 \mathrm{log}$ reduction in HIV RNA plasma viral $\operatorname{load}^{13}$; it also has a synergistic activity with nucleoside analogues such as zidovudine which may account for the survival benefit in comparison with ganciclovir (12.6 months versus 8.5 months; $p=0.007$ ) observed in the SOCA trial. ${ }^{14}$

\section{Ganciclovir}

Ganciclovir is a nucleoside analogue which requires to be phosphorylated in CMV infected cells. Mutations of thymidine kinase as modifications of UL97 gene are main causes of ganciclovir resistance which develops over time during prolonged ganciclovir treat- ment. Mutation on DNA polymerase has also been reported with cross resistance with cidofovir. ${ }^{1516}$

Ganciclovir exists as two formulations: intravenous ganciclovir represents the standard treatment given as $5 \mathrm{mg} / \mathrm{kg}$ twice daily as induction therapy for 14 to 21 days, and 5 $\mathrm{mg} / \mathrm{kg}$ one daily as maintenance treatment; oral ganciclovir is hampered by a low bioavailability (5\%) leading to use of a high daily dosage ( $3 \mathrm{~g} /$ day given as a $1 \mathrm{~g}$ regimen three times daily). Its lesser efficacy than intravenous formulation gives a median time of progression to $\mathrm{CMV}$ retinitis measured by retinal photographs of about 55 days compared with 65 days with the intravenous formulation. This difference was not statistically significant, but what was significant was the progression rate when observed by funduscopic examination ( 75 days with oral ganciclovir and 100 days with intravenous ganciclovir $\left.{ }^{17}{ }^{18}\right)$. The use of oral ganciclovir is limited to maintenance therapy and to primary prophylaxis of CMV retinitis.

The most limiting adverse reactions for ganciclovir are haematotoxicity, mainly neutropenia and anaemia. Growth factors such as G-CSF/GM-CSF may be used in patients with severe neutropenia when a switch to foscarnet is not possible.

Development of resistance of CMV to ganciclovir is correlated with the duration of drug exposure. In a study of 16 viral isolates from patients treated for more than a month, the percentages of both high level and low level ganciclovir resistance were respectively $19 \%$ and $81 \%$ when treatment was less than 9 months and $64 \%$ and $36 \%$ when treatment was longer than a year. Most of this resistance was correlated with the appearance of a mutation within the UL97 gene which encodes for the CMV specific transferase. Furthermore, high level ganciclovir resistance has a strong correlation with cidofovir resistance; some isolates of highly ganciclovir resistant strains might develop foscarnet resistance more quickly than expected. ${ }^{19}$ In brief, these data mean that the development of viral resistance is correlated with antiviral treatment which allows persistent viral growth; ganciclovir resistance is common over long term treatment and may induce cross resistance to other drugs.

\section{Cidofovir}

Cidofovir (Vistide HPMPC) is a nucleotide analogue of cytosine with potent in vitro and in vivo activity of prolonged duration against a broad spectrum of herpes viruses including CMV and herpes simplex type 1 and 2, VZV, and Epstein-Barr virus (EBV). Unlike aciclovir or ganciclovir which require intracellular activation by viral encoded enzyme, conversion of cidofovir to cidofovir diphosphate, which is the active cellular metabolite, is performed by host cellular enzymes and is therefore independent of viral infection. The diphosphate has a long intracellular half life (17-65 hours) which results in prolonged antiviral effects of cidofovir. The major dose 
limiting toxicity is dose dependent nephrotoxicity leading to degeneration and necrosis of renal proximal tubule cells. Concomitant administration of probenecid is thought to reduce the toxicity by competing with cidofovir for uptake at the surface of the tubule thereby minimising cidofovir toxicity, although the pharmacokinetics is complicated and probably irrelevant at the lower dose of 3 $\mathrm{mg} / \mathrm{kg}$.

Cidofovir is administered in acute therapy as $5 \mathrm{mg} / \mathrm{kg}$ once a week for 2 weeks, followed by $5 \mathrm{mg} / \mathrm{kg}$ every 2 weeks as maintenance therapy, and prehydration with a 1 litre intravenous infusion of normal saline. Oral probenecid is given as a $2 \mathrm{~g}$ dose in the 3 hours before infusion, and $1 \mathrm{~g}, 2$ and 8 hours after infusion.

When there is concomitant use of probenecid, zidovudine clearance decreases by $50 \%$, so patients should be advised to decrease by $50 \%$ or interrupt zidovudine use on each day of cidofovir injection.

In a prospective controlled trial ${ }^{20}$ comparing immediate treatment with cidofovir $(5 \mathrm{mg} / \mathrm{kg}$ weekly) with other treatments in non-sight threatening retinitis, the median time to progression of retinitis was 22 days compared with 120 days for the immediate treatment group $(p<0.0001)$. In this study, proteinuria occurred in $12 \%$ of patients, elevation of serum creatinine in $5 \%$ of patients; overall, $24 \%$ of patients discontinued treatment because of nephrotoxicity. Renal toxicity may not always be reversible. Early immunological symptoms of renal tubular necrosis such as hypophosphoraemia, hypokalaemia, and hypoglycaemia should be looked for. Patients with pre-existing renal dysfunction or mild proteinuria should avoid the use of cidofovir. After two or three cidofovir treatments $50-60 \%$ of patients had evidence of mild to moderate probenecid reactions, most commonly consisting of fever, chills, and rash. ${ }^{2021}$

Cidofovir is currently licensed for the treatment of CMV retinitis and in Europe for patients failing standard therapy.

Although cidofovir is not an easy drug to manage, because of its potential side effects, the major advantage is the possibility for maintenance therapy to be given as one injection every 2 weeks. The impact on quality of life, especially as the patient does not need an indwelling central catheter, compared with daily administrations of foscarnet or ganciclovir is of importance.

\section{Lobucavir}

Lobucavir is a guanine nucleoside analogue with a broad spectrum antiviral activity against CMV, herpes simplex, and VZV; interestingly, the compound has also in vitro inhibiting activity on HIV replication in monocytemacrophage cell line, probably through inhibition of reverse transcriptase activity and it appears also to have in vitro activity against hepatitis B. Bioavailability following oral administration of lobucavir varies around $50 \%$. $^{22} 23$

Phase I/II studies of lobucavir have demon- strated an in vivo activity on CMV treatment in urine or semen. Phase III trials in treatment and prophylaxis of retinitis are planned.

\section{Adefovir}

Adefovir (PMEA) is a nucleotide analogue of adenosine with activity on a broad spectrum of retro and herpes viruses including $\mathrm{HIV}$ and CMV. ${ }^{24} 25$ Phosphorylation of PMEA in cells to PMEApp is independent of virus infection; thus, PMEA may prime uninfected cells to resist viral infection when subsequently infected. Because of low oral bioavailability, several compounds have been synthesised to circumvent this problem; among them adefovir dipivoxil (bis POM PMEA) has an advantageous pharmacokinetic profile. Preliminary data suggest that adefovir leads to a $0.6 \log _{10}$ reduction in HIV viral load and 0.3 to $0.7 \log _{10}$ decrease in CMV viral load when studied in a small number of patients. A large phase III placebo controlled trial with adefovir both as anti-HIV and prophylactic drug is being planned.

\section{Prodrug of ganciclovir}

Valganciclovir is the valine ester of ganciclovir, a prodrug that is de-esterified to produce ganciclovir in the peripheral blood $\mathrm{A}$ dose ranging study $^{26}$ in $32 \mathrm{HIV}$ positive patients $-460 \mathrm{mg}$, $875 \mathrm{mg}, 1750 \mathrm{mg}, 2625 \mathrm{mg}$ once daily-has shown that the bioavailability of the drug is approximately $60 \%$ and increased by $20 \%$ with food absorption. A single daily dose of $900 \mathrm{mg}$ would achieve concentrations that are equivalent to a dosage of $5 \mathrm{mg} / \mathrm{kg} /$ day intravenous ganciclovir. ${ }^{26}$ Further studies to evaluate clinical efficacy of valganciclovir in induction and maintenance therapy of CMV retinitis are in progress.

\section{LOCAL THERAPY}

The rationale for using local therapy is to administer a specific treatment at the site of the infectious process, thus avoiding all the complications of systemic therapy. Several local treatment strategies have been used over time.

\section{Intravitreal injections}

Ganciclovir has been the drug used most often. Intravitreal injections with ganciclovir include one injection twice weekly as induction therapy followed by one injection weekly as maintenance therapy $(400 \mu \mathrm{g}$ ganciclovir per injection). Although no randomised studies have compared intravitreal injections with intravenous ganciclovir, results demonstrate a similar efficacy of the two treatments for progression in the affected eye.

\section{Intravitreal foscarnet}

There have been fewer experiences with intravitreal injections of foscarnet which is not easy to infuse given the large volume needed.

\section{Intravitreal implants}

An important approach has been the concept of permanent intravitreal devices to deliver ganciclovir continuously over several months 
(up to 6-8 months). These intraocular ganciclovir implants have been evaluated in two randomised studies in comparison with intravenous ganciclovir. Better control of CMV retinitis was obtained with ganciclovir implants $(2 \mu \mathrm{g} / \mathrm{h})$ with a median time of progression of 180-200 days; a higher risk of extraocular disease (15\%) was usually observed and the development of CMV retinitis in the fellow eye, with $50 \%$ of cases within 6 months. ${ }^{27} 28$

The main advantages of local therapy, particularly with intraocular ganciclovir implants, is a better control of CMV retinitis, compared with systemic intravenous infusion and a major improvement in quality of life avoiding any systemic potentially toxic therapy. However, the major drawback of this local therapeutic strategy is to treat only the eye affected by CMV and not the whole CMV pathological process, with the consequence of development of retinitis in the fellow eye or occurrence of extraocular CMV complications. Other specific complications of local therapy include conjunctival bleeding and infection; retinal detachment, a common complication in the long term CMV retinal process, might be facilitated by regular injections.

MANAGEMENT OF CMV RETINITIS THERAPY

One particular feature of CMV retinitis is its high tendency to relapse. Because of an overall longer survival, patients may experience several acute CMV retinitis episodes. Therefore, it is important to adapt to each individual clinical situation and give the optimal therapy. The acute treatment of CMV retinitis necrosis optimally consists of 2 or 3 weeks of intravenous treatment. The choice between foscarnet and ganciclovir will depend on various factors such as concurrent or previous anti-CMV treatment, renal function, the polymorphonuclear cell count and haemoglobin level, the current clinical and social status of the patient, and any concomitant antineoplastic chemotherapy.

Reasons to choose ganciclovir are ease of administration, and a good clinical tolerance, whereas reasons to prefer foscarnet are a proved or possible resistant CMV strain to ganciclovir, neutropenia or low haemoglobin level, a specific anti-HIV effect that may complement antiretroviral therapy, and the absence of need for expensive leucocyte growth factors.

In cases of relapsing retinitis, it might be wise to change the treatment, especially if time to relapse has been short, suggesting a lesser sensitivity of the virus. It has been demonstrated that a combination of foscarnet and ganciclovir was more effective in halting progression of CMV retinitis in patients with multiple CMV retinitis episodes. ${ }^{29}$

Local therapy, mainly with intravitreal implants, may be used in the absence of systemic CMV disease.

\section{CMV prophylaxis}

The ideal management of any infectious disease is a preventive strategy, particularly in infections with severe morbidity. Therefore, unsurprisingly, there have been different approaches to study the possibility of primary prophylaxis of CMV infection.

\section{ORAL GANCICLOVIR}

Two randomised placebo controlled phase III studies have been completed, ${ }^{30}{ }^{31}$ with controversial results. In study 1654 from Roche, oral ganciclovir ( $3 \mathrm{~g}$ per day) led to a $50 \%$ reduction in the risk of CMV disease compared with placebo with a occurrence rate of CMV retinitis in the ganciclovir group of $24 \%$ compared with $49 \%$ after 18 months. $^{5}$

The CPCRA study (994 patients) had planned a different management strategy, where retinal lesions were not systematically looked for and funduscopic examination performed only after occurrence of visual symptoms; after a 12 month median time of follow up, there was no significant difference regarding the occurrence of CMV disease in the oral ganciclovir group (11\%) compared with the placebo group $(12 \%)$; one suggested hypothesis for the difference observed has been the protective role of DDI on CMV while combination of DDI and oral ganciclovir might have a negative effect. ${ }^{31}$

Overall these data suggest that the prophylactic role of $\mathrm{CMV}$ is far from being optimal and, given the benefit-risk ratio, it appears necessary to better define the population of HIV infected patients who might benefit the most from prophylaxis. Interestingly, in study 1654, the analysis of CMV viral load measured by PCR $^{5}$ in correlation with the occurrence of CMV visceral disease shows that:

- a positive viral load was correlated to the occurrence of CMV disease;

- patients who were CMV PCR positive at baseline had a CMV disease rate of $26 \%$ in the ganciclovir group compared with $43 \%$ in the placebo group $(p=0.017)$. However, in patients with CMV viral load greater than 50000 copies, there was no efficacy of ganciclovir compared with placebo (75-100\% CMV events in each group).

- the best prophylactic index was obtained in patients with negative CMV PCR at entry, where the rate of CMV disease was $1 \%$ in the ganciclovir group compared with $14 \%$ in the placebo $(p<0.001)$; however, the low risk of CMV in this group decreases the interest in any prophylactic regimen which is potentially toxic, represents a high number of pills (12/day), and is expensive. Oral ganciclovir is the only drug approved for prophylaxis of $\mathrm{CMV}$ retinitis in patients with less than $100 \mathrm{CD} 4$ lymphocytes $\times$ $10^{6} / 1$.

\section{VALACICLOVIR}

Valaciclovir is the prodrug of aciclovir and allows plasma concentrations equivalent to that observed with aciclovir. A large phase III study $^{32}$ had evaluated valaciclovir $(2 \mathrm{~g} \times 4$ per day) versus two dosages of aciclovir (high dose $800 \mathrm{mg} \times 4$ per day/low dose $400 \mathrm{mg} \times 4$ per day). Overall, the valaciclovir group experienced less retinitis and less CMV organ dis- 
eases. The time to confirm CMV disease was significantly longer in the valaciclovir arm that in the two aciclovir groups. However, there was a trend $(p=0.06)$ towards earlier mortality in the valaciclovir group compared with low dose aciclovir but not compared with high dose aciclovir for which, to this date, the reason remains unknown. Furthermore, an unusual number of patients with haemolytic uraemic syndrome/thrombotic thrombocytopenic purpura was observed with valaciclovir. Further work is required to identify a dose of valaciclovir which is better elevated while maintaining a CMV protective effect and which provides a survival rate similar to that of aciclovir. For all these reasons, valaciclovir is not, in practice, used as prophylactic agent in HIV.

\section{Conclusion}

The control of CMV infection is a major challenge given the morbidity/mortality of this viral disease in $\mathrm{HIV}$ infection. In the past few years, important progress has been made regarding diagnosis, predictive virological markers, and quantification of CMV viral load for a better understanding of CMV disease; investigation of new drugs, with broad viral spectrum of activity, will be of importance for a better clinical management of patients with immune suppression.

1 Gallant JE, Moore RD, Richman DD, Keruly J, Chaisson $R E$. Incidence and natural history of cytomegalovirus disease in patients with advanced human immunodeficiency virus disease treated zidovurine. The Zidovudine Epidemiology Study. Group. $\mathcal{F}$ Infect Dis 1992;166: 1223-7.

2 Peters BS, Beck EJ, Anderson S, Coleman D, Coker R, Main J, et al. Cytomegalovirus infection in AIDS patients. Patterns of disease, response to therapy and patients. Patterns of disease, response to
trends in survival. 7 Infect 1991;23:129-37.

3 Salmon D, Lacassin F, Harzic F, Leport C, Perronne C, Bricaire F, et al. Predictive value of cytomegalovirus viremia for occurrence CMV organ involvement in AIDS. F Med Virol 1990;32:150-63.

4 Hansen KK, Rickstein A, Hoffman B, Nomild B, Olofsson $S$, Mathiesen L, et al. Detection of cytomegalovirus DNA in serum correlates with clinical cytomegalovirus in AIDS. $\mathcal{Y}$ Infect Dis 1994;170:1271-4.

5 Spector SA, Pilcher M, Lamy P, et al. PCR of plasma for cytomegalovirus DNA identifies HIV-infected persons most likely to benefit from oral ganciclovir prophylaxis most likely to benefit from oral ganciclovir prophylaxis

6 Rasmussen L, Morris S, Zipeto D, Fessel J, Wolitz R, Dowling A, et al. Quantitative of human cytomegalovirus DNA from peripheral blood cells of human immunodeficiency virus-infected patients could predict cytomegalovirus retinitis. F Infect $D$ is 1995;171:177-82.

7 Oberg B. Antiviral effects of phosphonoformate (PFA, foscarnet sodium). Pharmacol Ther 1989;40:213-85.

8 Ganem D, HHMI and UCSF. KSJ/HHV8 and Kaposi's sarcoma 1997; 4th Conference on Retroviruses and sarcoma 1997; 4th Conference on Retroviruses and
Opportunistic Infections. Washington, 22-26 January (abstract S24-S44).

9 Jacobson MA, Causey D, Polsky B, Hardy D, Chown M, Davis $\mathrm{R}$, et al. A dose-ranging study of daily maintenance intravenous foscarnet therapy for cytomegalovirus retinitis in AIDS. F Infect Dis 1993;168:444-8.

10 Katlama C, Dohin E, Caumes E, Cochereau-Massin I, Brancon C, Robinet $M$, et al. Foscarnet induction therapy for cytomegalovirus retinitis in AIDS: comparison of twice-daily and three-daily regimens. $₹$ AIDS 1992;5 (Suppl):S18-S24.

11 Deray G, Martinez F, Katlama C, Levaltier B, Beaufils H, Danis $\mathrm{M}$, et al. Foscarnet nephrotoxicity: mechanism, incidence and prevention. Am $\mathcal{f}$ Nephrol 1989;9:316-21.

12 Benson P, Nahass R, Deresinski S, et al. Safety of oral versus intravenous hydratation during induction therapy with intravenous foscavir (TM) in AIDS patients with CMV infections 1997. 4th Conference on Retroviruses and Opportunistic Infections. Washington, 22-26 January (abstract 299-S31).

13 Kaiser L, Perrin L, Hirshel B, Furrer H, Von Overbeck J, Olman $M$, et al. Foscarnet decreases human immunodeficiency virus RNA. F Infect Dis 1995;172:225-7.

14 Studies of Ocular Complications of AIDS Research Group in collaboration with AIDS Clinical Trials Group. Mortality in patients acquired immunodeficiency syndrome treated with either foscarnet or ganciclovir for cytomegalovirus retinitis. $N$ Engl $f$ Med 1992;326: cytomegalor. 20 .

15 Stanat SC, Reardon JE, Erice JA, Jordan MC, Drew WL, Biron KK. Severe resistant cytomegalovirus clinical isolates. Mode of resistance to ganciclovir. Antimicrob Agents Chemother 1991;35:2191-7.

16 Drew WL, Miner RC, Bisch DF, Follansbee SE, Gullett J, Mehalko SG, et al. Prevalence of resistance in patients receiving gangiclovir for serious cytomegalovirus infection. F Infect Dis 1991;163:716-9.

17 Drew WL, Ives D, Lalezari JP, Crumpacker C, Follansbee $\mathrm{SE}$, Benson CA, et al. Oral ganciclovir as maintenance treatment for cytomegalovirus retinitis in patients with treatment for cytomegalovirus retinitis
AIDS. $N$ Engl $\mathcal{f}$ Med 1995;333:615-20.

18 Oral Ganciclovir European and Australian Cooperative Study Group. Intravenous versus oral ganciclovi European/Australian cooperative study of efficacy and safety in the prevention of cytomegalovirus retinitis recurrence in patients with AIDS. AIDS 1995;9:471-7.

19 Spector SA. Cytomegalovirus: advances in pathogenesis and improved approaches for treatment and prevention 1997. 4th Conference on Retroviruses and Opportunistic 1997. 4th Conference on Retroviruses and Opportunistic
Infections. Washington, 22-26 January (abstract L3-S36).

20 Lalezari JP, Kemper C, Stagg RJ, et al. A randomized, controlled study and efficacy of intravenous cidofovir (CDV HPMPC) for the treatment of relapsing cytomegaloviru retinitis in patients with AIDS. XI Int Conf AIDS, 1996; Vancouver (abstract ThB304).

21 Lalezari JP, Stagg RJ, Kupperman BD, Holland GN, Kremer F, Ives DV, et al. Intravenous cidofovir for peripheral cytomegalovirus retinitis in patients with peripheral cytomegalovirus retinitis in

22 Flaherty J, Lalezari J, Petty B, et al. Pharmacokinetics and safety of oral lobucavir in cytomegalovirus-infected HIV patients. 4th Conference on Retroviruses and Opportunistic Infections. Washington, 22-26 January 1997; (abstract 302-S31).

23 Lalezari J, Drew L, Jordan C, et al. In vivo anti-CMV activity and safety of oral Lobucavir in HIV-infected patients 1997. 4th Conference on Retroviruses and Opportunistic 1997. 4th Conference on Retroviruses and Opportunistic 301S31)

24 Pauwels R, Balzarini J, Schols D, Baba M, Desmyter J, Rosenberg I, et al. Phosphonylmethoxyethyl purine derivatives, a new class of antiviral human immunodeficiency virus agent. Antimicrob Agents Chemother 1988;32 1025-30.

25 Balzarini J, Naesens L, Slachmuylders J, Niphuis $H$, Rosenberg I, Holy A, et al. 9-(2-Phosphonylmethoxyethy) adeninel (PMEA) effectively inhibits retrovirus replication in vitro and Simian immunodeficiency virus replication in vitro and Simian immunodeficiency
virus infection in rhesus monkeys. AIDS 1991; virus $5: 21-8$.

26 Brown F, Arum I, Francis G, et al. Ganciclovir prodrug (RS-79070)-multiple dose, dose-ranging study with effect of food 1997. 4th Conference on Retroviruses and Opportunistic Infections. Washington, 22-26 January (abstract LB19-S88)

27 Martin DF, Parks DJ, Mellow SD, Ferris FL, Walton RC Remaley NA, et al. Treatment of cytomegalovirus retinitis with an intraocular sustained-release ganciclovir implant. with an intraocular sustained-releas

28 The Chiron Ganciclovir Implant Study Group. A randomized controlled multicenter clinical trial of a sustainedreleased intraocular ganciclovir implant in AIDS patients with CMV retinitis. 35th Interscience Conference on Antimicrobial Agents and Chemotherapy. 1995. San Francisco, abstract 215

29 Studies of Ocular Complication of AIDS Research Group AIDS Clinical Group. Combination foscarnet and ganciclovir therapy vs monotherapy for the treatment of clovir therapy vs monotherapy for the treatment of
relapsed cytomegalovirus retinitis in patients with AIDS. relapsed cytomegalovirus retinitis

30 Spector SA, McKinley GF, Lalezari JP, Samo T, Andruczk $\mathrm{R}$, Follansbee $\mathrm{S}$, et al. Oral ganciclovir for the prevention of cytomegalovirus disease in persons with AIDS. $N$ Engl f Med 1996;334:1491-7.

31 Brosgart CL, Craig C, Hillman D, et al. Final results from randomized, placebo-controlled trial of the safety and efficacy of oral ganciclovir for the prophylaxis of CMV retinal and gastrointestinal mucosal disease 1996. XI Int Conf AIDS, Vancouver (abstract ThB301).

32 Feindberg J, Cooper D, Hurwitz S, et al. Phase III study of valaciclovir (VACV) for cytomegalovirus (CMV) prophylaxis in patients with advanced HIV disease 1995. 35th Interscience Conference on Antimicrobial Agents and Chemotherapy, San Francisco (abstract I112). 